\title{
COMPOSICION QUIMICA Y DIGESTIBILIDAD DE INSUMOS ALIMENTICIOS DE LA ZONA DE UCAYALI
}

\author{
J ulio M. R osales* \\ Tony T ang**
}

\section{RESUMEN}

EI presente estudio se realizó en el Centro Regional de Investigación del IIAP, filial Ucayali. El objetivo fue determinar la composición química y la digestibilidad de 22 insumos alimenticios regionales.

Para la determinación de la composición química se aplicó el método de W eende a fín de determinar: proteína bruta, fibra cruda, extracto etéreo, cenizas y nifex.

Para la prueba de digestibilidad se utilizó la técnica de digestibilidad in vitro.

En base a los resultados obtenidos, se pueden clasificar los insumos, según su contenido de nutrientes y grado de digestibilidad, en: Insumos proteicos: de buena digestibilidad: (harina de pescado "boquichico"); de regular digestibilidad (harina de follaje de yuca); y de baja digestibilidad (harina de sangre). Insumos energéticos: de buena digestibilidad (maíz amarillo, polvillo de arroz, nielen de arroz, harina de yuca, harina de cáscara de yuca, harina de yuca + cáscara, afrecho de yuca, harina de plátano, y harina de plátano + cáscara); de regular digestibilidad ( harina de cáscara de plátano, y harina de kudzu; y de baja digestibilidad (orujo de cervecería). Insumos fibrosos: de buena digestibilidad (harina de hoja de amasisa); de regular digestibilidad (harina de hoja de plátano, harina de centrosema, y harina de stylo); y de baja digestibilidad (harina de coronta de maíz, harina de cáscara de cacao y harina de desmodio).

\footnotetext{
* Investigador del área pecuaria del Centro Regional de Investigación del Instituto de Investigaciones de la A mazonía Peruana, filial U cayali (CRI-IIA P-U cayali).

** Tesista del área pecuaria de la U niversidad Nacional de U cayali (UNU).
} 


\section{INTRODUCCION}

El elevado costo de los insumos alimenticios tradicionales, usados en la alimentación animal, ocasiona una baja rentabilidad en la actividad pecuaria, ya que los costos de alimentación, en animales monogástricos, representa entre el 60 y $75 \%$ del costo total de producción. U na de las alternativas para corregir esta limitante es el uso de recursos alimenticios regionales, tales como productos agrícolas, residuos de cosecha, subproductos agroindustriales y leguminosas forrajeras.

Para formular un alimento balanceado, de acuerdo a la especie y la clase animal, es necesario conocer el valor nutricional de los insumos, especialmente de aquellos insumos regionales no tradicionales, que cuentan con gran potencial para la alimentación animal.

El objetivo del presente estudio fue determinar la composición química y la digestibilidad de 22 insumos alimenticios regionales.

\section{REVISION BIBLIOGRAFICA}

Insumo alimenticio. Church, (1987); afirma que el insumo alimenticio es la materia prima fundamental para la producción animal. Actualmente existe una diversidad de insumos alimenticios, variando según el lugar. Se han clasificado más de dos mil insumos diferentes sin contar las variedades de forrajes y granos que se proporciona a los animales, y que no son consumidos por el hombre o los que abundan en exceso en un determinado lugar.

Cordova, (1993); menciona que los alimentos pueden clasificarse de acuerdo a una serie de criterios. Estos pueden ser en base a su origen, en base a su concentración de nutrientes y finalmente en base a su empleo.

La clasificación en base a su contenido de nutrientes se considera su concentración en proteína, energía y fibra, y se dividen principalmente en tres grupos: 
- Insumos Proteicos Son aquellos que contienen 20\% o más de proteína y menos de $18 \%$ de fibra bruta en base seca.

- Insumos energéticos son aquellos que contienen menos de $20 \%$ de proteina y menos de $18 \%$ de fibra cruda en base seca.

- Insumos fibrosos son aquellos que se caracterizan por tener una baja concentración de nutrientes, de digestibilidad variable, generalmente baja y por un al to contenido de fibra, que varía entre 18 y $35 \%$ en base seca.

Análisis Proximal. M aynard et al, (1989) indican que el análisis proximal o de Weende, de los alimentos, es el esquema químico más empleado para describir los alimentos, y comprende seis fracciones: humedad, extracto etéreo, proteína cruda, cenizas, fibra cruda y extracto no nitrogenado (nifex), correspondiendo la suma de los dos últimos al total de los carbohidratos del alimento.

Digestibilidad. Según Flores (1986) la digestibilidad comprende todos los procesos que sufren los alimentos en el tracto digestivo, desde la masticación y la mezcla de los alimentos con la saliva en la boca, digestión, descomposición química y la absorción de nutrientes, así como la expulsión de los materiales no digeridos a través del ano.

En general, existen dos métodos para determinar la digestibilidad en las diferentes especies animales. Estos son: digestibilidad in vivo y digestibilidad In Vitro. En el primer método se utiliza directamente el animal, mientras que en el segundo, se trata de reproducir en el laboratorio en la forma más simplificada, los procesos de digestión, aunque a veces se usa el animal en forma parcial 0 indirecta.

Cordova (1993) afirma que el método in vitro es más rápido y más económico. Se trata de un método que trata de reproducir en el laboratorio las funciones de los microorganismos del rumen, sometiendo al alimento, finamente triturado y molido, a diferentes acciones de ácidos, bases y enzimas.

\section{MATERIALES Y METODOS}

Ubicación y Duración del Estudio. El estudio se realizó entre los meses de marzo y agosto de 1992, en la Estación Experimental "Villa Rica" del Centro 
Regional de Investigación del IIAP, filial U cayali. Geográficamente esta situada a 8022' 13" latitud Sur, 740 34' 23" longitud Oeste, y a una altitud de 154 msnm.

Area de muestreo. A barcó la zona de Pucallpa y lugares al edaños, según la natural eza de cada muestra.

- Las muestras de productos y subproductos agrícolas se colectaron directamente del fundo del agricultor y los subproductos agroindustriales de los centros donde se procesan.

- L Las muestras de forrajes, se colectaron de potreros del IVITA, 60 días después del último corte.

- Las otras muestras, se colectaron del centro de abastos de Pucallpa (pescado boquichico), del camal de Pucallpa (sangre de vacuno), y de los campos de la UNU (hoja de amasisa y hoja de cético).

A condicionamiento de las $M$ uestras. $L$ as muestras se secaron a una temperatura de 60 a $70 \stackrel{\circ}{C}$ durante 48 horas. Luego fueron molidas en un molino de martillo, embolsadas y etiquetadas, para su análisis en el laboratorio de Nutrición de la U niversidad Nacional A graria de la Selva.

\section{Variables Medidas}

Composición Q uímica. Para determinar la composición química de los insumos, se realizó un análisis usándose el método de W eende (M uñoz y M endoza, 1990), mediante el cual se evalúan los siguientes componentes:

a) Humedad. Se determinó por el método de secado en una estufa al vacío a $105^{\circ} \mathrm{C}$ por 24 horas.

b) Proteína Cruda. Se midió por el método de micro kjeldhal, que comprende tres fases: digestión, destilación y titulación.

c) Extracto Etereo. Se determinó por el método de extracción por hexano 0 método Soxhlet. 
d) Fibra Cruda. Se obtuvo eliminando los carbohidratos solubles por hidrólisis a compuestos más simples (azúcares), mediante la acción de los ácidos y alcalis débiles en caliente.

e) Ceniza. Se determinó eliminando la materia orgánica por calcinación a 600 $\stackrel{\circ}{ }$.

f) Extracto Libre de nitrógeno. Se obtuvo por diferencia, al restar 100 menos los resultados de proteína, extracto etéreo, fibra y ceniza.

Digestibilidad. Se realizó análisis de Digestibilidad in vitro usando la técnica de dos etapas: de Tilley y Terry (1968), que involucra primeramente un período de incubación de 48 horas con licor ruminal de ganado vacuno más saliva artificial y en segundo término la digestión en una mezcla de ácido clorhídrico y pepsina.

Componentes en Estudio. Se usaron 22 insumos:

- Maíz amarillo (Zea mays).

- Coronta de maíz.

- Polvillo de arroz común (Oriza sativa).

- Nielen de arroz.

- Y uca con cáscara (M anihot esculenta).

- Y uca sin cáscara.

- Cáscara de yuca.

- A frecho de yuca.

- Plátano con cáscara (M usa sp).

- Plátano sin cáscara.

- Hoja de plátano.

- Cáscara de plátano.

- Orujo de cervecería.

- Hoja de cetico (Cecropia sp).

- Hoja de amasisa ( Erythrina sp ).

- Sangre de vacuno.

- Pescado boquichico (Prochilodus niqricans).

- Cáscara de cacao (Theobroma cacao).

- Kudzú (Pueraria phaseoloides).

- Centrosema (Centrosema pubescens).

- Stylo (Stylosanthes guianensis).

- Desmodio (D esmodium ovalifolium). 


\section{RESULTADOS}

Cuadro 1. Análisis Proximal de los principales insumos alimenticios, en base seca $(\%)$

Insumo

Materia Proteína Grasa Fibra Ceniza Nifex seca cruda cruda cruda

$\begin{array}{lrrrrrr}\text { H arina maíz } & 88.4 & 10.02 & 6.69 & 3.07 & 1.43 & 78.79 \\ \text { H arina coronta de maíz } & 84.7 & 2.64 & 1.62 & 30.00 & 1.41 & 64.33 \\ \text { Polvillo de arroz } & 86.7 & 11.81 & 7.57 & 7.45 & 5.03 & 68.14 \\ \text { N ielen de arroz } & 86.7 & 11.86 & 4.13 & 3.55 & 2.56 & 77.90 \\ \text { Harina yuca } & 88.1 & 3.18 & 0.98 & 1.54 & 2.22 & 92.08 \\ \text { H na. cáscara de yuca } & 87.7 & 5.11 & 0.87 & 19.31 & 9.51 & 65.20 \\ \text { H na. yuca con cáscara } & 87.9 & 2.59 & 0.71 & 2.05 & 2.24 & 92.41 \\ \text { A frecho yuca } & 87.2 & 2.72 & 0.15 & 6.12 & 3.58 & 87.43 \\ \text { H na. hoja de yuca } & 87.5 & 25.75 & 6.92 & 10.95 & 6.05 & 50.33 \\ \text { H na. plátano } & 90.1 & 3.04 & 0.71 & 0.36 & 1.93 & 93.96 \\ \text { H na. cáscara de plátano } & 88.7 & 5.93 & 4.51 & 10.63 & 12.07 & 66.86 \\ \text { H na. plátano con cáscara } & 87.8 & 3.95 & 1.44 & 2.03 & 3.09 & 89.49 \\ \text { H na. de hoja de plátano } & 87.2 & 12.70 & 10.28 & 24.38 & 12.60 & 40.04 \\ \text { Harina de sangre } & 86.2 & 80.47 & 0.33 & 1.62 & 2.78 & 14.80 \\ \text { H na. pescado boquichico } & 87.9 & 55.56 & 16.72 & 1.51 & 17.90 & 8.31 \\ \text { H na. cáscara de cacao } & 88.9 & 10.56 & 9.61 & 24.23 & 11.88 & 43.72 \\ \text { Orujo cervecería } & 87.4 & 16.42 & 6.70 & 9.39 & 3.83 & 63.66 \\ \text { Harina hoja de cetico } & 87.8 & 16.18 & 2.00 & 19.09 & 8.51 & 54.22 \\ \text { Harina hoja de amasisa } & 88.1 & 19.36 & 4.56 & 22.63 & 6.88 & 46.57 \\ \text { H na. K udzú } & 87.4 & 15.99 & 1.92 & 13.74 & 5.40 & 62.95 \\ \text { H na. C. pubescens } & 88.7 & 17.47 & 2.02 & 32.22 & 4.76 & 43.53 \\ \text { Harina Stylo } & 87.9 & 14.60 & 0.99 & 30.22 & 4.98 & 49.21 \\ \text { Harina D esmodio } & 89.1 & 12.08 & 1.01 & 33.92 & 4.26 & 48.73 \\ & & & & & & \end{array}$


Cuadro 2. Digestibilidad in vitro de los principales insumos alimenticios regionales $(\%)$

M uestra en estudio

H na. maíz

H na. coronta de maíz

Polvillo de arroz

$\mathrm{N}$ ielen de arroz

Harina de $\mathrm{Y}$ uca

H arina cáscara de $Y$ uca

H arina Y uca con Cáscara

A frecho de $Y$ uca

Harina de hoja de yuca

Harina de plátano

H arina de cáscara Plátano

H arina de Plátano + cáscara

Harina hoja de plátano

$\mathrm{H}$ arina de sangre

Harina pescado Boquichico

H arina cáscara de cacao

Orujo de cervecería

$\mathrm{H}$ arina hoja cetico

H arina hoja de amasisa

H arina de kudzú

$\mathrm{H}$ arina de centrosema

$\mathrm{H}$ arina de stylo

$\mathrm{H}$ arina de desmodio
Digestibilidad

materia seca
Digestibilidad

materia orgánica
58.59

21.96

64.89

58.15

81.53

69.13

74.24

84.81

53.13

82.08

45.49

72.91

40.60

39.49

68.79

31.46

37.95

30.28

53.25

46.82

41.20

59.34

34.45
58.67

21.73

64.21

57.73

82.08

74.44

74.25

85.21

51.76

82.19

38.47

72.55

35.34

38.42

62.90

23. 15

36.88

25.95

50.78

44.35

41.62

57.39

32.16 


\section{DISCUSION}

Maíz. En el maíz amarillo grano se encontró 10,02\% de proteína y buen contenido de nifex: $78,79 \%$. Mientras que la harina de coronta de maíz presentó bajo contenido de proteína (2,64\%) y alto contenido de fibra (30\%).

El contenido proteico del maíz grano fue superior a lo reportado por Huarcaya (1987) que es de 8,8\%, pero inferior a lo encontrado por Saldaña (1984) que es de 10,2\%. Estas diferencias se atribuyen principalmente a los siguientes factores: variedad, época de siembra y tipo de suelo.

La digestibilidad de la harina de maíz fue buena, debido a su alto contenido de carbohidratos solubles, fácilmente asimilables y a su bajo contenido de fibra. Por el contrario en la harina de coronta de maíz fue baja $(21,96 \%)$ debido a su alto contenido de fibra.

Subproductos del Arroz. El polvillo de arroz reportó un contenido de $11,51 \%$ de proteína y $68,14 \%$ de carbohidratos solubles; mientras que en el nielen presentó un contenido proteico de $11,86 \%$ y $77,90 \%$ de nifex. El valor de la proteína del polvillo es inferior a lo reportado por Saldaña (1984) de $12,8 \%$ y superior a lo encontrado por Carranza (1974) de 10,63 y García (1978) de $11,8 \%$. Mientras que para nielen el valor de proteína fue superior a lo reportado por Reyes (1991) de 9,7\%; éstas variaciones probablemente se deban a la variedad de arroz, al método de pilado, tipo de suelo y época de siembra.

La digestibilidad del polvillo y el nielen fueron buenas, debido al alto contenido de nifex y regular contenido proteico.

Productos de la Yuca. La harina de yuca con cáscara, el afrecho de yuca y la harina de yuca sin cáscara, mostraron menores contenidos de proteína, siendo respectivamente 2,$59 ; 2,72$ y 3,18\%. En cambio, la harina de la cáscara de yuca y la harina de follaje de yuca, reportaron mayores contenidos de proteína, siendo respectivamente 5,11 y $25,75 \%$. Sin embargo, su alto contenido de fibra, 19,31 y 10,95\%, respectivamente, limitan su uso en altas proporciones en la ración de monogástricos.

Resultados similares fueron reportados por Choque (1987) en la harina de yuca con 2,75\% de proteína, por Ríos (1974) en el afrecho de yuca con 
5,34\% de proteína, y por Carvalho y Kato, citado por Herrera (1989) en la harina de hoja de yuca con $25,42 \%$ de proteína.

La digestibilidad fue buena para la harina de yuca con cáscara, afrecho de yuca y la yuca sin cáscara, debido a sus al tas proporciones de carbohidratos solubles. Por el contrario, en la harina de cáscara de yuca y la harina de follaje de yuca, pese a sus mayores contenidos de proteína que los insumos anteriores, presentaron menores digestibilidades, debido posiblemente a sus al tos nivele de fibra.

Productos del Plátano. La harina de plátano solo y la harina de plátano con cáscara presentan buenos contenidos de carbohidratos solubles, pero sus bajos tenores proteicos limitan su utilización en altas proporciones en animales en fase de inicio o crecimiento. Por otro lado, principalmente la harina de follaje de plátano, seguida por la harina de cáscara de plátano presentan buenos niveles proteicos, 12,7 y 5,93\% respectivamente; pero sus altos niveles de fibra cruda, 24,38 y 10,63\%, limitan su utilización en altas proporciones en raciones alimenticias de animales monogástricos.

El valor proteico de la harina de plátano es inferior a lo reportado por Guevara (1980) y Reyes (1991) quienes encontraron, 3,69 y 3,75\%, respectivamente. Igualmente, los tenores proteicos de la harina de plátano con cáscara, harina de cáscara de plátano y harina de hoja de plátano fueron menores a lo reportado por Reyes (1991) quien encontró 4,01; 6,06 y $16,64 \%$, respectivamente.

El valor de la fibra cruda de la harina de hoja de plátano fue mayor a lo reportado por Dextre (1978) que fue de $21,2 \%$. Estas diferencias se podrían atribuir a factores como variedad, fertilidad del suelo y método de procesamiento.

La digestibilidad fue buena, tanto para la harina de plátano solo como para la harina de plátano con cáscara, debido principalmente a su elevado contenido de carbohidratos solubles.

M ientras que las harinas de cáscara de plátano y hoja de plátano, reportaron menores digestibilidades como consecuencia a sus al tas proporciones de fibra cruda. 
Guevara (1980) reportó un resultado similar de digestibilidad de la materia seca de la harina de plátano $(81,71 \%)$, mientras que Dextre (1978) encontró un valor superior $(79,04 \%)$.

Harina de Sangre. La harina de sangre de vacuno presenta buen contenido proteico $(80,47 \%)$, superior a lo reportado por Huarcaya (1987) con $65,6 \%$, debido posiblemente a factores como método de preparación y tipo de secado. Sin embargo, la digestibilidad fue baja, debido posiblemente a su bajo nivel de carbohidratos solubles.

Harina de Pescado Boquichico. La harina de pescado boquichico presenta buenos contenidos de proteína, grasa y cenizas (Cuadro 1). Resultados de contenidos proteicos superiores fueron reportados por Cortez y Sánchez (1991), siendo 71,83\%, debido posiblemente al método de secado y preparación.

L a digestibilidad, tanto de la materia seca como de la materia orgánica fue buena, siendo 68,79 y $62,90 \%$, respectivamente. Debido a su al to contenido de carbohidratos solubles.

Harina de Cáscara de Cacao. La harina de cáscara de cacao presenta regular contenido proteico $(10,56 \%)$ y altos contenidos de ceniza y fibra cruda. Resultados con menores contenidos de proteína y fibra cruda fueron reportados por Vicuña (1974) con 6,44 y $21,73 \%$, respectivamente; y A Imeida (1978) con 7,85 y $23,78 \%$, respectivamente.

L a digestibilidad fue baja, como consecuencia de su al to nivel de fibra cruda. Siendo inferior a lo reportado por V icuña (1974) quien encontró 93,54\% de digestibilidad de materia seca, mediante la técnica in vivo en ovinos.

Orujo de Cervecería. El orujo de cervecería seco muestra un buen contenido de proteína $(16,42 \%)$ y carbohidratos solubles $(63,66 \%)$, que sumado a su moderado contenido de fibra cruda $(9,39 \%)$ lo perfila como un insumo de gran utilidad.

El valor proteico obtenido es inferior a los reportado por García (1978) de $20,20 \%$; estas variaciones se atribuye al grano, método de obtención y natural eza de los aditivos empleados. 
La digestibilidad fue regular, debido a su moderado contenido de fibra cruda. Tolentino (1978) encontró valores superiores de digestibilidad (65,95\%) utilizando la técnica in vivo en ovinos.

Harina de Hoja de Cetico. La harina de hoja de cetico presenta un buen contenido proteico $(16,18 \%)$ y regulares niveles de carbohidratos solubles $(54,22 \%)$. Sin embargo, su alto contenido de fibra $(19,09 \%)$ limita su utilización en altas proporciones en animales monogástricos. Rosado (1974) reportó un resultado con menor tenor proteico $(13,67 \%)$, la que podría deberse principalmente a la edad de la planta.

La digestibilidad, tanto de la materia seca como de la materia orgánica fue baja, inferior a lo reportado por Rosado (1974) de 73,88\%, debido posiblemente a la técnica in vivo empleada.

Harina de Hoja de Amasisa. La harina de hoja de amasisa presenta un buen contenido de proteína $(19,36 \%)$, moderado contenido de carbohidratos solubles $(46,57 \%)$ y un alto contenido de fibra cruda $(22,63 \%)$, valores que lo hacen un insumo de regular calidad nutritiva. Guerra (1988) reportó un valor ligeramente superior en cuanto a proteína $(20,4 \%)$.

La digestibilidad de la materia seca y la materia orgánica obtenidas fueron buenas, 53,25 y $50,78 \%$, respectivamente, debido a su buen contenido de proteína y moderado nivel de carbohidratos solubles.

Harina de Leguminosas Forrajeras. Entre las leguminosas forrajeras, el kudzu fue la que reportó mayor cantidad de carbohidratos solubles, menor contenido de fibra y regular contenido proteico. Con relación al contenido de proteína, las que mejores cantidades reportaron en orden decreciente fueron centrocema, kudzu, stylo y desmodio.

Valores superiores de proteína para kudzu, fueron reportados por Sánchez (1972) con 19,73\%, Arana (1973) con 22,76\%, y Gonzales (1976) con $17,35 \%$.

Reyes (1991), encontró menor contenido proteico en stylo (12\%); en cambio, en desmodio encontró un valor superior (12,42\%).

Las mejores digestibilidades de las harinas de leguminosas, en orden decreciente, fueron para stylo, centrocema y kudzu. La que reportó menor 
digestibilidad fue el desmodio, esto se debió principalmente a su menor proporción de proteína y alta proporción de fibra cruda.

Sánchez (1972) encontró mejor digestibilidad de la materia seca $(63,86 \%)$ para el kudzu, empleando la técnica in vivo en ovinos.

\section{CONCLUSIONES}

1. De los resultados obtenidos en el presente estudio podemos clasificar a los insumos regional es estudiados en:

Insumos proteicos: a) de buena digestibilidad: harina de pescado "boquichico"; b) de regular digestibilidad: harina de follaje de yuca; y c) de baja digestibilidad: harina de sangre.

Insumos energéticos: a) de buena digestibilidad: maíz amarillo, polvillo de arroz, nielen de arroz, harina de yuca, harina de cáscara de yuca, harina de yuca + cáscara, afrecho de yuca, harina de plátano, y harina de plátano + cáscara ; b) de regular digestibilidad: harina de cáscara de plátano y harina de kudzu; c) de baja digestibilidad: orujo de cervecería.

Insumos fibrosos: a) de buena digestibilidad: harina de hoja de amasisa; b) de regular digestibilidad: harina de hoja de plátano, harina de centrocema y harina de stylo; c) de baja digestibilidad: harina de coronta de maíz, harina de cáscara de cacao y harina de desmodio.

2. Los insumos regionales constituyen una alternativa para rebajar costos de producción en la alimentación de especies monogástricas en nuestra Región.

3. Son necesarios adicionales ensayos, con un mayor número de insumos a fin de aprovechar óptima y convenientemente los recursos regionales. 


\section{BIBLIOGRAFIA}

ALMEIDA, C.A. 1973. Sustitución parcial del maíz por harina de cáscara de cacao (Teobroma cacao) en la alimentación de pollos parrilleros. Tesis Ingeniero Zootecnista, Tingo M aría (Perú): U niversidad Nacional A graria de la Selva. 43 pp.

ARANA, A.R. 1973. Sustitución de maíz por harina de plátano en la alimentación de pollos parrilleros. Tesis Ingeniero Zootecnista. Tingo M aría (Perú): U niversidad N acional A graria de la Selva. 51 pp.

CARRANZA， J. 1974. Digestibilidad del polvillo de arroz en ovinos. Tesis Ingeniero Zootecnista. Tingo María (Perú): Universidad Nacional A graria de la Selva. 62 pp.

CHOQUE, J. 1987. Efecto de diferentes niveles de harina de yuca (Manihot esculenta) en la alimentación de pollos de carne. Tesis Ingeniero Zootecnista. Tingo M aría (Perú): Universidad Nacional Agraria de la Selva. 55 pp.

CHURCH D. C. 1987. Fundamentos de nutrición y alimentación de animales. México: Ed. LIMUSA. 533 pp.

CORDOVA A., P. 1993. Alimentación A nimal. Lima (Perú): Editorial EDITEC. $244 \mathrm{pp}$.

CORTEZ, J. y SANCHEZ, H. 1991. Principales peces de consumo de la A mazonía Peruana. Iquitos (Perú): Instituto de Investigación de la A mazonía Peruana (IIAP). Dirección de Recursos Hidrobiológicos.

DEXTRE, R. 1978. Digestibilidad de la hoja de plátano en ovinos. Tesis Ingeniero Zootecnista. Tingo María (Perú): Universidad Nacional A graria de la Selva. 58 pp.

FLORES, J. 1986. Manual de Alimentación A nimal. Primera edición. M exico: Editorial Ciencia y Técnica. Mexico. D.F. 4 vols. 1096 pp.

GONZALES, A. C. 1976. Alimentación de Cobayos (Cavia cobayo) empleando asociación de gramineas y leguminosas en Tingo María. 
Tesis Ingeniero Zootecnista. Tingo M aría (Perú): U niversidad N acional A graria de la Selva. 74 pp.

GARCIA, G. 1978. Utilización de diferentes niveles de residuos de cervecería en raciones de crecimiento y engorde de pollos parrilleros. Tesis Ingeniero Zootecnista. Tingo María (Perú): Universidad Nacional Agraria de la Selva. 60 pp.

GUERRA, M. A. 1988. Alimentación de Cobayos (Cavia cobayo con forraje de erytrina (Erythrina $\mathrm{sp)}$ ) suplementado con diferentes niveles de concentrado comercial. Tesis Ingeniero Zootecnista. Tingo M aría (Perú): U niversidad Nacional A graria de la Selva. 69 pp.

GUEVARA, M. 1980. Digestibilidad del plátano verde (Musa sp) en ovinos. Tesis Ingeniero Zootecnista Tingo María (Perú): Universidad Nacional A graria de la Selva. 63 pp.

HERRERA, E. M. 1989. Utilización de la harina de Hoja de Yuca (Manihot esculenta) en la alimentación de pollos de carne en el trópico. Tesis Ingeniero Zootecnista. Tingo María (Perú): U niversidad Nacional A graria de la Selva. 52 pp.

HUARCAYA, H.A. 1987. Valor Nutricional de la harina de sangre de broilers en el trópico. Tesis Ingeniero Zootecnista. Tingo María (Perú): Universidad N acional A graria de la Selva. 59 pp.

MAYNARD, L. A. et al., 1989. Nutrición A nimal. 7ma. edición. M éxico: Editorial Mc GRAW-HILL. 640 pp.

MUÑOZ, A. M. y MENDOZA, M.E. 1990. Manual de prácticas de nutrición I. Lima (Perú): U niversidad Nacional Agraria La Molina. Departamento de Nutrición. 34 pp.

REYES, J. 1991. Composición de Insumos no tradicionales usados en la alimentación animal en la provincia de Piura. Piura: U niversidad $\mathrm{N}$ acional de Piura. Facultad de Zootecnia. 17 pp.

RIOS, B.R. 1974. Digestibilidad de los elementos nutritivos del afrecho de yuca en ovinos. Tesis Ingeniero Zootecnista. Tingo M aría (Perú):U niversidad N acional A graria de la Selva. 67 pp. 
ROSADO, T.R. 1974. Digestibilidad de la hoja de cético (Cecropia sp) en ovinos. Tesis. Ingeniero Zootecnista. Tingo María (Perú): Universidad Nacional A graria de la Selva. 69 pp.

SALDAÑA, M.A. 1984. Utilización de diferentes niveles de harina de cáscara de cacao (Theobroma cacao) en raciones para cuyes (Cavia cobayo) en engorde. Tesis Ingeniero Zootecnista. Tingo María (Perú):U niversidad Nacional A graria de la Selva. 71 pp.

SANCHEZ, R.A. 1972. Digestibilidad del kudzu tropical (Pueraria phaseoloides) en ovinos. Tesis Ingeniero Zootecnista. Tingo María (Perú):U niversidad N acional A graria de la Selva. 68 pp.

TILLEY, J.; TERRY, R. 1968. Procedure for the in vitro digestion of herbage samples. Hurley England. The Grassland Research Institute. Bull

VICUÑA, D. A. 1974. Digestibilidad de la cáscara de cacao en ovinos. Tesis Ingeniero Zootecnista. Tingo M aría (Perú): Universidad Nacional Agraria de la Selva. 57 pp. 